\title{
The effect of vitamin Con the gene expression profile of sperm protamines in the male partners of couples with recurrent pregnancy loss: A randomized clinical trial
}

\author{
Saeideh Hamidian ${ }^{1}$, Ali Reza Talebi ${ }^{2,3}$, Farzaneh Fesahat ${ }^{4}$, Mohammad Bayat $^{1}$, Ali Mohammad Mirjalili' ${ }^{1}$, Hamid Reza Ashrafzadeh ${ }^{3}$, \\ Mahya Rajabi ${ }^{5}$, Fateme Montazeri ${ }^{3}$, Saeid Babaei ${ }^{1}$ \\ 'Department of Anatomy, Faculty of Medicine, Arak University of Medical Sciences, Arak; ${ }^{2}$ Department of Anatomy, Faculty of Medicine, ${ }^{3} Y a z d$ \\ Reproductive Sciences Institute, and ${ }^{4}$ Reproductive Immunology Research Center, Shahid Sadoughi University of Medical Sciences, Yazd; ${ }^{5}$ Genetics \\ Department of Biology, Yazd Science and Arts University, Yazd, Iran
}

Objective: Since sperm abnormalities are known to be a major reason for recurrent pregnancy loss (RPL), any defects in DNA structure and chromatin condensation can place embryos at risk in the early stage of development and implantation. As antioxidants such as vitamin C may play a protective role against the destruction of protamine genes in sperm chromatin, this study was conducted to evaluate the effects of vitamin C on chromatin and the expression of protamine genes in the male partners of couples with RPL.

Methods: Twenty male partners of couples with RPL were selected as the intervention group and received vitamin C supplementation ( 250 mg daily for 3 months). Healthy fertile men $(n=20)$ were included as controls. Sperm chromatin, DNA integrity, and the expression levels of protamine genes were evaluated before and after treatment.

Results: Significant differences were found in sperm morphology, protamine deficiency, and apoptosis between the two groups and before and after vitamin C administration. A significant change was found in mRNA levels of $P R M 1, P R M 2$, and the PRM1/PRM2 ratio after treatment.

Conclusion: Daily oral administration of vitamin C may improve human sperm parameters and DNA integrity by increasing protamine gene expression levels in the male partners of couples with RPL. The beneficial effects of vitamin C supplementation as an antioxidant for the male partners of couples with RPL could lead to improved pregnancy outcomes in these cases.

Keywords: Clinical trial; Gene expression; Protamines; Recurrent pregnancy loss; Spermatozoa; Vitamin C

\section{Introduction}

Recurrent pregnancy loss (RPL) is a major concern in reproductive medicine [1]. It is defined as more than two clinical pregnancy losses

Received: July 22, 2019 Revised: September 16, 2019.

Accepted: September 18, 2019

Corresponding author: Saeid Babaei

Department of Anatomy, Faculty of Medicine, Arak University of Medical

Sciences, Arak 3819693345, Iran

Tel:+98-9124941241 Fax:+98-8633133147 E-mail:saeid.babaei@yahoo.com

*This study was extracted from the MSc thesis of Saeideh Hamidian.

*This study was financially supported by Yazd Institute for Reproductive Sciences,

Yazd, Iran.

This is an Open Access article distributed under the terms of the Creative Commons Attribution Non-Commercial License (https://creativecommons.org/licenses/by-nc/4.0/) which permits unrestricted non-commercial use, distribution, and reproduction in any medium, provided the original work is properly cited. before the 20th week of gestation [2]. Various causes of RPL have been proposed, including immunological and hormonal disorders and/or genetic anomalies such as chromosomal abnormalities in embryos [3]. A recent descriptive case-control study concluded that unexplained RPL may be due to an increased frequency of abnormal sperm parameters, sperm DNA fragmentation (SDF), defective chromatin condensation, or sperm aneuploidy [4]. A recent systematic review and meta-analysis of 15 prospective studies suggested a significant association between SDF and RPL [5]. The aforementioned review showed a remarkably higher rate of SDF in the male partners of women with RPL than was found in the male partners of fertile women, with a mean difference of 11.91 (95\% confidence interval, 4.97-18.86) [5].

There are two protamine genes in humans that are located on 
chromosome 16: PMN1, which codes for protamine P1, and PMN2, which codes for protamine P2 [6]. In human sperm cells, $85 \%$ of the histones are replaced by protamine, allowing sufficient chromatin density to be achieved [7] and reducing the availability of sites that could be harmed by DNA-damaging agents [8]. Evidence indicates that a high proportion of histones in sperm relative to protamine causes defective chromatin compaction, subsequently leading to an increased risk of male infertility [9]. A proper ratio of protamine P1 to protamine $\mathrm{P} 2$ (normal, 0.8-1.2) is required for normal sperm function [10]. The inappropriate expression of PMN1 and/or PMN2 [11], both at the mRNA and protein level [12], can lead to an abnormal ratio of protamine P1 to protamine P2 in infertile men. Furthermore, some reports have demonstrated that changes in the genes coding for protamines modulated substantial changes in sperm chromatin structure, resulting in failed spermatogenesis $[13,14]$.

Sperm abnormalities, such as DNA or/and chromatin damage, can endanger the early stage of embryo development and the potential of embryos for implantation [15]. Lack of chromatin integrity, which may manifest as protamine deficiency, can influence sperm function [7] and quality [16]. Moreover, protamine deficiency can interfere with DNA protection due to the distinct importance of protamineDNA complex stability [17].

In the human body, antioxidant systems are present in order to neutralize free radicals [18]. An imbalance between free radical production and the antioxidant capacity causes oxidative stress (OS), which affects the structure, function, and motility of spermatozoa, leading to male infertility $[19,20]$. Antioxidant-based dietary supplementation promotes the motility of sperm cells and appropriate sperm concentrations [19,21].

Vitamin C is a water-soluble substance that is absorbed through the small intestine [22]. Previous studies showed that effective antioxidants, such as vitamin C, are essential for the maintenance of sperm function and DNA integrity [20,23]. Moreover, vitamin C deficiency is associated with OS induction in the male reproductive system, which leads to reduced sperm production [24].

Notwithstanding the potential role of vitamin $C$ in improving human sperm quality, no prospective trial has yet assessed the effect of vitamin $C$ on the expression of protamine genes. Therefore, the aim of this randomized clinical trial (RCT) was to examine whether taking at least $250 \mathrm{mg}$ of vitamin $\mathrm{C}$ daily for 90 days improved sperm parameters and the gene expression profile of protamines in the male partners of couples with RPL.

\section{Methods}

\section{Participant selection}

This prospective study was registered in the Iranian Registry of Clin- ical Trials with the national ID No. IRCT20180312039059N1. Informed consent was obtained from all participants. The participants were randomly selected among patients referred to the Yazd Research and Clinical Center for Infertility using a random number table. Twenty male partners of couples with RPL due to male factor infertility were selected as the intervention group. The control group $(n=20)$ included the male partners of couples with no varicocele, no chronic diseases, a normal genital examination, normal sperm parameters according to the 2010 World Health Organization (WHO) criteria [25], and a child born in the last 2 years. The inclusion criteria for participants were as follows: age of less than 40 years, no history of alcohol abuse or use of any types of tobacco and/or cigarettes, no antidepressant use, a sperm concentration of 7-14 million/mL, total sperm motility $<40 \%$, and a percentage of sperm with normal morphology $<4 \%$. Obesity (based on body mass index), diabetes, and varicocele disorders were exclusion criteria. None of the included couples had been treated with antioxidants or other medications before this study. Men who were treated with other medications during the study were also excluded. In addition, a number of exclusion criteria were defined for the female partners, including the absence of a hormonal imbalance, chromosomal alterations, tubal obstruction, and bacterial or viral infections.

The intervention group was administered $250 \mathrm{mg}$ of vitamin C daily in tablet form (Avicenna Co., Tehran, Iran) for 3 months by an expert urologist. The dosage of vitamin $C$ and the duration of the intervention were set in accordance with a previous report on the efficient duration and dosage of vitamin C treatment [26]. The participants were recommended to consume fruits and vegetables regularly during their treatment, and to refrain from soft drinks, soybeans, any kind of canned food, and even unnecessary use of mobile phones and/or laptops. After 3 months, sperm parameters and chromatin were assessed in the intervention group. Moreover, pregnancy outcomes were followed in the couples who received vitamin C supplementation. All procedures performed in studies involving human participants were in accordance with the ethical standards of the institutional and/or national research committee and with the 1964 Helsinki Declaration and its later amendments or comparable ethical standards. This study was approved by the Ethics Committee of Yazd Research and Clinical Center for Infertility. In this study, all procedures were conducted by an experienced laboratory technician who was blinded to the study to the allocation of participants.

\section{Hormone assessments}

In order to ensure the reproductive health of the female partners of couples in the intervention group and to facilitate comparisons with the controls, levels of sex hormones in the female partners were measured at the beginning of the study. Blood samples $(5 \mathrm{~mL})$ were 
collected from all participants. Blood fractionation was done by 10 minutes of centrifugation at 2,500 $\times$ g. Ultimately, $3 \mathrm{~mL}$ of the upper phase (blood serum) was separated using special enzyme-linked immunosorbent assays to measure levels of follicle-stimulating hormone (FSH), luteinizing hormone (LH), prolactin (PRL), and anti-Müllerian hormone $(\mathrm{AMH})$.

\section{Sperm sample collection}

Semen samples were obtained by masturbation after at least 4872 hours of sexual abstinence. Semen analysis was performed according to the $2010 \mathrm{WHO}$ guidelines [27]. The specimens were pre-

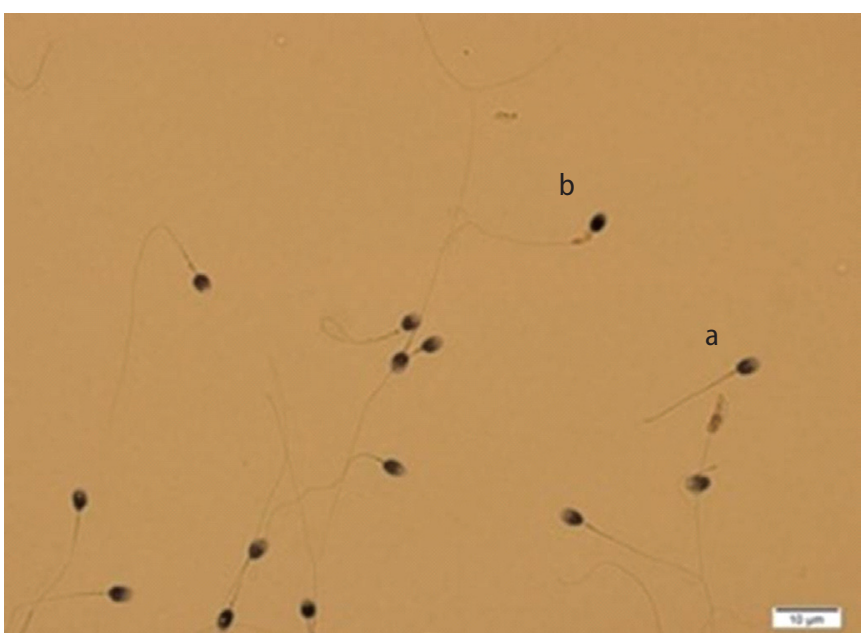

Figure 1. Visualization of sperm morphology using the Diff-Quick staining method. a, sperm with normal morphology; b, abnormal sperm morphology $(60 \times 47 \mathrm{~mm} ; 120 \times 120 \mathrm{DPI})$. Magnification, $\times 1,000$.

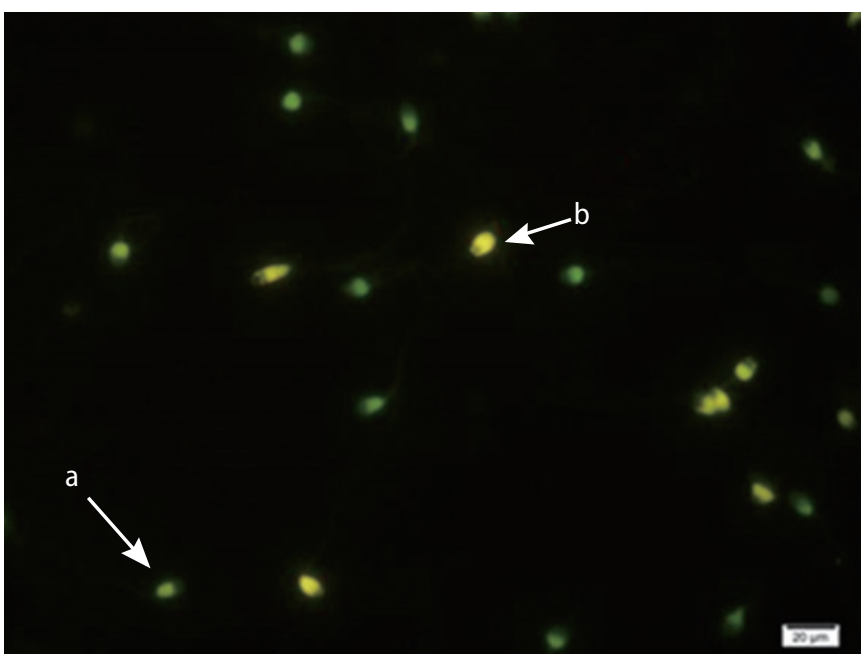

Figure 2. Assessment of sperm protamine deficiency using chromomycin A3 (CMA3) staining. a, normal chromatin seen as dark yellow; b, CMA3+ protamine shown as bright yellow $(70 \times 57 \mathrm{~mm} ; 120 \times 120$ DPI). Magnification, $\times 1,000$. pared by the swim-up method. Half of the prepared specimens were used for morphological examinations and chromatin/DNA integrity assessments, and the other half was used to evaluate participants gene expression profile.

\section{Sperm parameters}

The analysis of all samples was conducted according to the WHO standard values provided in 2010 [25]. Sperm concentration and motility were determined under light microscopy at $\times 400$ magnification. The Diff-Quik staining method was used to evaluate sperm morphology. At least 200 sperm cells were counted in each slide with a magnification of $\times 1,000$ (Figure 1 ).

\section{Sperm DNA integrity tests \\ 1) Sperm protamine deficiency}

To assess sperm protamine deficiency, Carnoy solution was used to fix each sample slide for 10 minutes at $4^{\circ} \mathrm{C}$. Subsequently, each slide was treated with chromomycin A3 (CMA3) solution for 10 minutes in a darkroom (Sigma-Aldrich, Sigma-Aldrich, MO, USA) and analyzed under fluorescent microscopy ( $\times 1,000$ magnification) (Figure 2) [28]. Spermatozoa with protamine defects $(\mathrm{CMA} 3+)$ were stained as bright yellow, and normal spermatozoa (CMA3-) as dark yellow.

\section{2) Sperm DNA fragmentation}

Sperm apoptosis was quantified through a terminal deoxynucleotidyl transferase dUTP nick-end labeling (TUNEL) assay using a kit (In Situ Cell Death Detection Kit with Fluorescein; Roche, Indianapolis, IN, USA). Spermatozoa with normal DNA were visualized as white, while

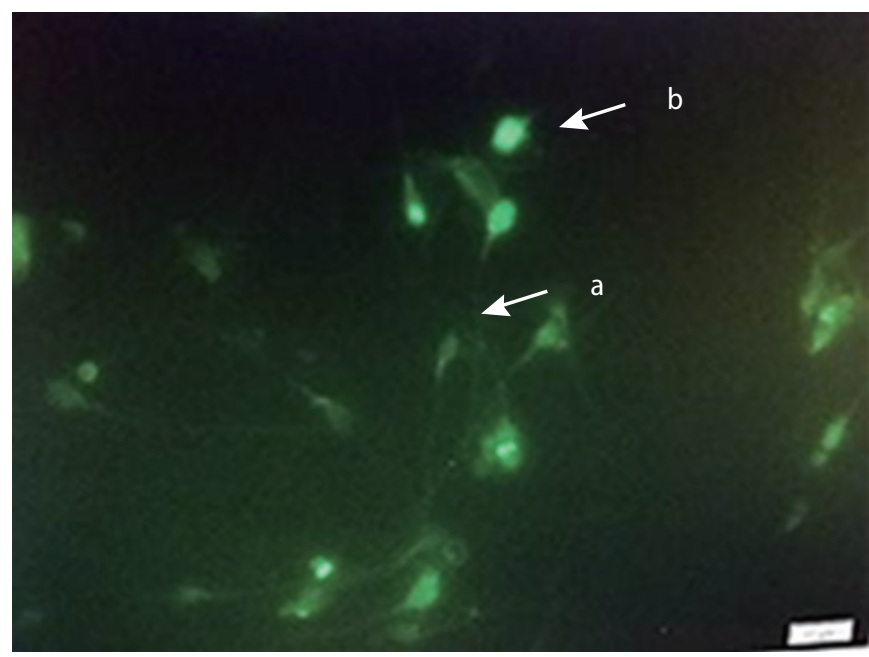

Figure 3. Evaluation of apoptosis in sperm by a TUNEL assay. a, sperm with normal DNA; b, sperm with high DNA fragmentation (TUNEL+; $93 \times 43$ mm; $120 \times 120$ DPI). TUNEL, terminal deoxynucleotidyl transferase dUTP nick-end labeling. 
sperm with a strong fluorescent color demonstrated increased levels of SDF (Figure 3) [27,28].

\section{Protamine gene expression profile}

Total RNA extraction and cDNA synthesis of all sperm samples were performed using an RNeasy Plus Universal Mini Kit (Qiagen, Hilden, Germany) and a cDNA synthesis kit (Applied Biosystems, Foster City, $C A, U S A)$, respectively, following the manufacturer's instructions. RNA concentration and purity were detected by a NanoDrop spectrophotometer (Thermo Scientific, Waltham, MA, USA). The adjusted concentration of purified total RNA (100 ng/ $/ \mathrm{L}$ ) was applied for cDNA

Table 1. Oligonucleotide primers

\begin{tabular}{llll}
\hline Accession No. & Gene & \multicolumn{1}{c}{ Primer sequence (5'-3') } & $\begin{array}{c}\text { PCR } \\
\text { product } \\
(\mathrm{bp})\end{array}$ \\
\hline NM_002761.2 & PMN1 & F: AGA GCC GGA GCA GAT ATT ACC & 119 \\
& & R:TCT ACA TCG CGG TCT GTA CCT & \\
NM_002762.3 & PMN2 & F: ATC CAC AGG CGG CAG CATC & 144 \\
& & R:TTC CAG CTG GGG GTG AGG GG & \\
NM_004048.2 & B2M & F: AGA TGA GTA TGC CTG CCG TG & 106 \\
& & R:TGC GGC ATCTCAAACCTC & \\
\hline
\end{tabular}

$\mathrm{PCR}$, polymerase chain reaction; $P M N 1$, protamine $\mathrm{P} 1 ; \mathrm{PMN} 2$, protamine $\mathrm{P} 2$, $B 2 M$; beta-2-microglobulin; F, forward; $R$, reverse. synthesis. The relative gene expression levels of PMN1 and PMN2 were evaluated by quantitative real time-polymerase chain reaction (ABI 7300; USA) utilizing SYBR Green PCR Master Mix (Applied Biosystems). Beta-2 microglobulin (B2M) was used as the reference gene. Table 1 summarizes the primers used for all the evaluated and controlled genes (PMN1, PMN2, and B2M). The $2^{-\triangle \Delta C T}$ technique was used to present levels of gene expression normalized to those of B2M using threshold cycle (CT) values. In this method, $\Delta C T$ is defined as the

Table 2. Comparison of clinical data between the intervention and control groups before treatment with vitamin C

\begin{tabular}{lrrc}
\hline Hormone & \multicolumn{1}{c}{ Case } & Control & $p$-value \\
\hline FSH $(\mathrm{mlU} / \mathrm{mL})$ & $11.92 \pm 0.39$ & $13.35 \pm 0.78$ & 0.111 \\
$\mathrm{LH}(\mathrm{mlU} / \mathrm{mL})$ & $25.37 \pm 3.16$ & $28.43 \pm 4.09$ & 0.557 \\
$\mathrm{PRL}(\mathrm{mlU} / \mathrm{mL})$ & $10.9 \pm 0.83$ & $12.9 \pm 1.15$ & 0.167 \\
AMH $(\mathrm{ng} / \mathrm{mL})$ & $2.38 \pm 0.14$ & $2.82 \pm 0.18$ & 0.580 \\
\hline
\end{tabular}

Values are presented as mean \pm standard error, and $p$-values $<0.05$ were considered to indicate statistical significance.

Case, female couples with recurrent pregnancy loss in the intervention group, in whom the relevant sex hormones were measured at the beginning of the study; Control, female couples of normozoospermic men, in whom the relevant sex hormones were measured at the beginning of the study; $\mathrm{FSH}$, follicle-stimulating hormone; LH, luteinizing hormone; PRL, prolactin; $\mathrm{AMH}$, anti-Müllerian hormone.

Table 3. Clinical characteristics of couples with recurrent pregnancy loss

\begin{tabular}{|c|c|c|c|c|c|c|}
\hline \multirow{2}{*}{$\begin{array}{l}\text { Patient } \\
\text { No. }\end{array}$} & \multicolumn{3}{|c|}{ Before treatment } & \multicolumn{3}{|c|}{ After treatment ( $3 \mathrm{mo})$} \\
\hline & $\begin{array}{l}\text { Duration of } \\
\text { infertility (yr) }\end{array}$ & $\begin{array}{c}\text { No. of } \\
\text { abortions }\end{array}$ & $\begin{array}{l}\text { Cycle } \\
\text { (N/A) }\end{array}$ & $\begin{array}{c}\text { Clinical pregnancy } \\
(+/-)\end{array}$ & $\begin{array}{l}\text { Live birth } \\
(+/-)\end{array}$ & $\begin{array}{c}\text { Abortion } \\
(+/-)\end{array}$ \\
\hline 1 & 4 & 2 & $\mathrm{~N}$ & + & + & - \\
\hline 2 & 9 & 2 & A & + & + & - \\
\hline 3 & 12 & 3 & $\mathrm{~N}$ & + & $-^{\text {a) }}$ & - \\
\hline 4 & 10 & 2 & A & + & + & - \\
\hline 5 & 8 & 2 & A & + & - & + \\
\hline 6 & 12 & 5 & A & + & + & - \\
\hline 7 & 7 & 3 & A & + & Ongoing & - \\
\hline 8 & 10 & 3 & A & - & - & - \\
\hline 9 & 5 & 2 & A & + & - & + \\
\hline 10 & 6 & 3 & $\mathrm{~N}$ & + & Ongoing & - \\
\hline 11 & 7 & 2 & A & + & Ongoing & - \\
\hline 12 & 5 & 3 & A & - & - & - \\
\hline 13 & 4 & 2 & A & - & - & - \\
\hline 14 & 8 & 2 & A & + & - & + \\
\hline 15 & 10 & 3 & - & - & - & - \\
\hline 16 & 8 & 2 & - & - & - & - \\
\hline 17 & 12 & 3 & A & + & - & + \\
\hline 18 & 9 & 2 & - & - & - & - \\
\hline 19 & 5 & 2 & A & + & + & - \\
\hline 20 & 11 & 3 & A & + & + & - \\
\hline
\end{tabular}

$\mathrm{N}$, natural cycle; $\mathrm{A}$, assisted reproductive technology cycle.

a) Stillbirth 
CT of a target gene (here, PMN1 or PMN2) minus the CT of a reference gene (here, $B 2 M$ ). Then, $\Delta \Delta C T$ is defined as the $\Delta C T$ of a target sample minus the $\Delta C T$ of a reference sample.

\section{Statistical analysis}

All data were statistically analyzed using IBM SPSS ver. 23.0 (IBM Corp., Armonk, NY, USA), with $p$-values $<0.05$ considered to indicate significant differences. The paired $t$-test was used to analyze the data before and after the treatment. One-way analysis of variance and the Tukey test were used to analyze the differences in gene expression levels within and between the groups. The Pearson test was also used to calculate correlations.

Table 4. Effects of vitamin C on sperm concentration and morphology in the intervention and control groups

\begin{tabular}{lccc}
\hline Variable & Intervention & Control & $p$-value \\
\hline $\begin{array}{l}\text { Concentration }\left(\times 10^{6} / \mathrm{mL}\right) \\
\text { Before treatment }\end{array}$ & $67.95 \pm 3.76$ & & \\
After treatment & $68.10 \pm 3.73$ & & 0.000 \\
$\quad p$-value & 0.97 & - & 0.000 \\
Morphology (\%) & & $8.75 \pm 0.35$ & - \\
$\quad$ Before treatment & $2.35 \pm 0.21$ & & 0.000 \\
After treatment & $6.35 \pm 0.67$ & & 0.51 \\
$p$-value & 0.000 & - & - \\
\hline
\end{tabular}

Values are presented as mean \pm standard error, and $p$-values $<0.05$ were considered to indicate statistical significance. Sperm parameters were evaluated according to the World Health Organization criteria (2010) [25]. The paired $t$-test, analysis of variance, and the Tukey test were used as appropriate given the data distribution.

Intervention, male partners of women with recurrent pregnancy loss; Control, normozoospermia men.

Table 5. Sperm chromatin and DNA evaluation in the intervention and control groups

\begin{tabular}{lccc}
\hline Variable & Intervention & Control & $p$-value \\
\hline $\begin{array}{l}\text { CMA3+ } \\
\text { Before treatment }\end{array}$ & $55 \pm 1.94$ & $24 \pm 0.01$ & 0.00 \\
After treatment & $22.40 \pm 0.96$ & & \\
$p$-value & 0.00 & - & 0.00 \\
TUNEL+ & & $7.4 \pm 0.99$ & \\
Before treatment & $20.2 \pm 0.97$ & & 0.65 \\
After treatment & $8.25 \pm 0.49$ & & 0.76 \\
$p$-value & 0.00 & - & \\
\hline
\end{tabular}

Values are presented as mean \pm standard error, and $p$-values $<0.05$ were considered to indicate statistical significance. The paired $t$-test and analysis of variance were used as appropriate given the data distribution.

Intervention, male partners of women with recurrent pregnancy loss; Control, normozoospermia men; CMA3, chromomycin A3; TUNEL, terminal deoxynucleotidyl transferase dUTP nick-end labeling.

\section{Results}

In this RCT, the male participants in the control and intervention groups were similar in terms of the demographic variables of age (33.5 \pm 1.1 vs. $35.1 \pm 1.5$ years) and body mass index ( $25.51 \pm 1.69$ vs. $25.75 \pm 1.25 \mathrm{~kg} / \mathrm{m}^{2}$ ). No complications were reported at the time of vitamin $\mathrm{C}$ administration during the study. Before starting the treatment with an antioxidant, the mean number of abortions and the duration of infertility in women with RPL were $2.3 \pm 0.95$ and $8.9 \pm 4.3$ years, respectively. Furthermore, all the women with RPL had hormonal levels in the normal range, including FSH, LH, PRL, and $\mathrm{AMH}$ (Table 2). As summarized in Table 3, pregnancy and live birth outcomes improved after treatment with vitamin $C$, while the number of abortions (20\%) showed a reasonable decrease in the women with RPL in the intervention group.

Sperm concentrations were significantly lower in the intervention group, both before and after treatment with vitamin C, than in the control group. However, after treatment, sperm concentrations improved in the intervention group compared to the values observed before treatment (Table 4). The proportion of normal sperm morphology was significantly lower in the intervention group than in the control group, before and after treatment. In contrast, we observed a significantly higher percentage of sperm morphology after treatment in the intervention group (Table 4). Compared to the control group, the proportion of sperm cells with protamine deficiency and

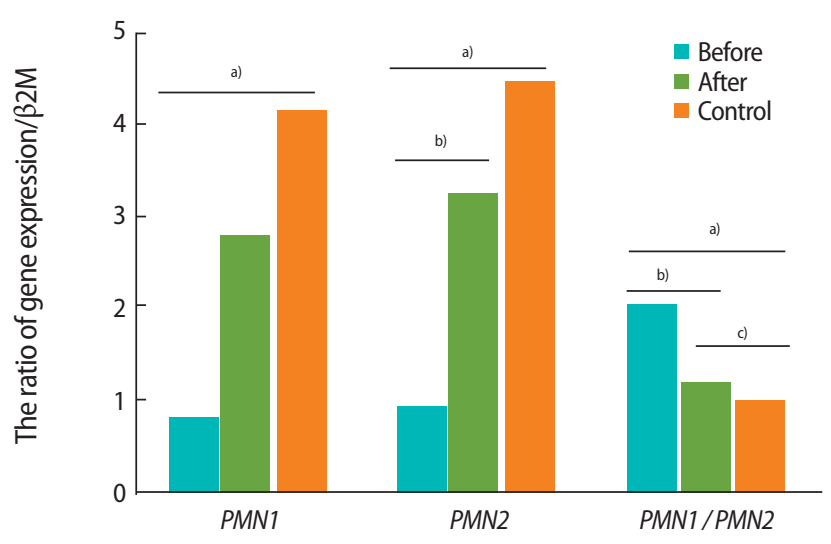

Figure 4. Comparison of relative levels of expression of protamine genes between the intervention and control groups. Values are presented as mean \pm standard deviation, and $p$-values $<0.05$ were considered to indicate statistical significance. The paired $t$-test and analysis of variance were used. Intervention, male partners of women with recurrent pregnancy loss; Control, normozoospermic men. ${ }^{a}$ Significant difference between the intervention group before vitamin $C$

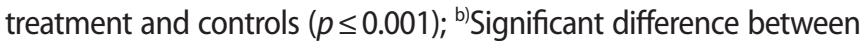
before and after treatment with vitamin C (PMN2: $p=0.04$ and PMN1/PMN2: $p=0.00)$; ${ }^{c}$ Significant difference between the intervention group after treatment with vitamin $C$ and controls $(p=0.00)$. 
Table 6. Correlations between sperm parameters, chromatin/DNA integrity, and protamine gene expression levels

\begin{tabular}{lll}
\hline Variable & PMN1 & PMN2 \\
\hline Concentration & $r=0.61$ & $r=0.17$ \\
& $p=0.61$ & $p=0.20$ \\
Normal morphology & $r=0.32$ & $r=0.43$ \\
& $p=0.01$ & $p=0.001$ \\
TUNEL (+) & $r=-0.30$ & $r=-0.38$ \\
& $p=0.02$ & $p=0.002$ \\
CMA3 (+) & $r=-0.31$ & $r=-0.37$ \\
PMN1 & $p=0.02$ & $p=0.003$ \\
& $r=1.00$ & $r=0.92$ \\
PMN2 & - & $p=0.000$ \\
& $r=0.92$ & $r=1.00$ \\
& $p=0.000$ & -
\end{tabular}

All nonparametric correlations were obtained using Pearson statistics, and $p$ values $<0.05$ were considered to indicate statistical significance.

PMN1, protamine 1; PMN2, protamine 2; TUNEL, terminal deoxynucleotidyl transferase dUTP nick-end labeling; CMA3, chromomycin A3.

apoptotic cells were remarkably higher in the intervention group before undergoing vitamin $C$ treatment. Both before and after the administration of vitamin $C$, a significant difference was present between the intervention and control groups with regard to the proportion of sperm cells with apoptosis and protamine defects. However, a reasonable decrease in the number of sperm cells with positive TUNEL and CMA3 findings was observed in the intervention group after the intervention (Table 5).

The gene expression levels of both PMN1 and PMN2 in the male partners of couples with RPL were significantly lower prior to treatment than those in the control group (by 3.9- and 4.6-fold, respectively. After taking vitamin C for 3 months, the PMN1/PMN2 ratio significantly decreased in the intervention group compared to the pretreatment values (Figure 4). As shown in Table 6, there were strong, significant, positive correlations between the expression levels of PMN1 and PMN2 $(r=0.92, p<0.001)$.

\section{Discussion}

To evaluate the possible impacts of vitamin $C$ on sperm parameters, chromatin and DNA integrity, and the protamine gene expression profile, 20 male partners of couples with RPL received $250 \mathrm{mg}$ of vitamin C daily for 3 months in the present study. Our results indicate that a diet rich in vitamin $\mathrm{C}$ could significantly improve sperm morphology to the normal range (normal morphology in more than 4\% of spermatozoa) in male partners (Table 4). Anbara et al. [29] also reported a positive association between vitamin $C$ intake and sperm quality. Gil-Villa et al. [30] investigated the effect of antioxidants on sperm parameters in male partners of couples with RPL, and report- ed that an antioxidant-rich diet could improve sperm parameters and function, in accordance with our findings. In contrast, the beneficial effects of antioxidants on sperm parameters were not confirmed by Ko and Sabanegh [31]. Akmal et al. [32] reported increases in the percentage of sperm with normal morphology and sperm concentration in infertile men after taking vitamin C orally $(1,000 \mathrm{mg}$ twice daily for 2 months). Okon and Utuk [33] suggested that oral administration of vitamin $C$ at moderate doses $(250 \mathrm{mg})$ significantly increased the quality of sperm parameters, serum levels of testosterone, and the percentage of normal sperm morphology. However, we did not observe any significant changes in sperm concentration. These differences can be attributed to the use of varying doses of vitamin C and short-term consumption in different studies [21].

However, we observed an impressive effect of vitamin C on sperm chromatin quality and DNA integrity (Table 5). It is thought that semen parameters do not directly represent the status of sperm nuclei, as sperm with normal motility and morphology may have chromatin and/or DNA abnormalities [34]. Previous studies have found significant positive correlations between DNA fragmentation and CMA3 positivity in infertile men $[27,28,35]$. Moreover, protamine defects were associated with the fertilization rate in stimulated assisted reproduction cycles according to Bianchi et al. [36] and Iranpour et al. [37]. Deemeh et al. [38] revealed that male partners of couples with RPL had high levels of protamine deficiency. As DNA integrity plays a key role in sperm function, it can be a better predictor of sperm fertility potential than conventional sperm parameters [39]. The results of three studies conducted by Aoki et al. showed that more severe protamine deficiency was related to DNA damage in infertile men $[11,14,40]$. Previous research demonstrated a positive correlation between reactive oxygen species levels and apoptosis rate in seminal plasma $[39,41]$. Antioxidants have been observed to reduce apoptosis in testicular cells and to improve sperm quality [42]. In addition, high levels of OS can change sperm function and chromatin structure, and can induce apoptosis by altering the amount of intracellular calcium [43]. Unlike oocytes, sperm cannot repair their DNA damage. Therefore, a sperm cell with damaged DNA is condemned to have poor-quality chromatin and functional deficiencies [44]. DNA damage is not considered to be the main cause of fertilization failure, but it is expected to impair embryo development and lead to the abortion of embryos [31].

In this RCT, we observed significantly lower expression levels of each protamine in the intervention group than in the control group, both before and after the intervention. We also discovered a negative correlation between PMN1 and PMN2 mRNA levels and sperm DNA integrity (Table 6). In line with our findings, Rogenhofer et al. [45] recently conducted an observational study and reported significant differences in PMN1 and PMN2 mRNA levels and the ratio of PMN1 to 
PMN2 mRNA between couples with recurrent miscarriages and healthy controls $(p<0.01)$. Despite the small sample size and ambiguity regarding the precise mechanism, they suggested that the levels of protamine gene expression in spermatozoa were not only essential for successful fertilization, but also for appropriate early embryo development [45]. In contrast, Torregrosa et al. [46] reported no relationship between PMN2 gene expression and sperm DNA integrity. Due to the associations of protamine replacement with acrosome formation and normal sperm morphology, any defects in the replacement process resulting from protamine deficiency (such as low protamine expression) may lead to sperm dysfunction, resulting in poor implantation and pregnancy outcomes [47]. We found that vitamin C could improve spermatogenesis by increasing protamine expression and chromatin condensation.

The relative expression of PMN2 significantly increased after vita$\min C$ treatment (Figure 4), explaining the higher ratio of PMN1 to $P M N 2$ in the intervention group than in the controls in this study. According to recent findings, the ratio of $P M N 1$ and $P M N 2$ expression is approximately 1:1 in healthy men, and any change in this ratio is associated with male infertility [10]. Another study showed that the $P M N 1 / P M N 2$ ratio was abnormal in infertile men [48]. Additionally, it has been found that changes in PMN2 expression leading to a reduction in the mRNA levels of PMN2 may be responsible for the altered proportion of $P M N 1$ to $P M N 2$ in infertile men [7].

It is believed that OS may have a destructive effect on fertility in both the male and female reproductive systems $[49,50]$. However, the presence of antioxidants in semen can protect chromatin and/or DNA integrity against free radical damage [51]. According to a recent study, vitamin C-along with some other antioxidants such as lycopene, coenzyme Q10, zinc, and selenium — can be used to treat OS in sperm. In the aforementioned report, a daily dose of $200 \mathrm{mg}$ or $1,000 \mathrm{mg}$ of vitamin C was recommended according to prescriptions designed specifically to augment male fertility [52]. Our clinical findings demonstrated improvements in pregnancy outcomes, including a lower rate of pregnancy loss and increased rates of clinical pregnancy and take-home babies (Table 3). In a case series, male partners were instructed to consume a diet rich in antioxidants or commercial multivitamins containing beta-carotene, vitamin $\mathrm{C}$, vitamin $\mathrm{E}$, and zinc for at least 3 months. In that study, all male partners of couples with a history of recurrent embryo loss who received antioxidant supplementation achieved successful pregnancy [30]. Vitamin C is known to be an important antioxidant for reducing sperm abnormalities and protecting sperm DNA from OS [29]. It is also required for normal spermatogenesis and sperm function [53]. Yun et al. [54] reported that vitamin C could significantly decrease lipid peroxidation and increase glutathione peroxidase levels. Consequently, in their study, it increased testosterone concentration and the sperma- tid population. The current RCT was the first attempt to investigate the potential impacts of antioxidants on protamine expression, at both the gene and protein levels, in the male partners of couples with RPL, and we suggest that further clinical trials should be carried out with larger populations and with assessments of the efficacy of other antioxidants.

In conclusion, our findings demonstrated a remarkable increase in protamine gene expression after treatment with vitamin C. It seems that daily oral administration of vitamin C can improve human sperm parameters and DNA integrity by increasing protamine gene expression levels in the male partners of couples with RPL. The beneficial effects of vitamin C supplementation as an antioxidant for the male partners of couples with RPL could lead to improved pregnancy outcomes in these cases.

\section{Conflict of interest}

No potential conflict of interest relevant to this article was reported.

\section{Acknowledgments}

The authors would like to thank the staff of the Andrology and Recurrent Abortion Research Center and the Research and Clinical Center for Infertility at Shahid Sadoughi University of Medical Sciences, Yazd, Iran

\section{ORCID}

Saeideh Hamidian $\quad$ https://orcid.org/0000-0003-3400-1389 Ali Reza Talebi Farzaneh Fesahat Mohammad Bayat https://orcid.org/0000-0003-4883-3020 https://orcid.org/0000-0002-3743-4449 https://orcid.org/0000-0003-1465-0015 Ali Mohammad Mirjalili Hamid Reza Ashrafzadeh Mahya Rajabi https://orcid.org/0000-0002-3456-5128 https://orcid.org/0000-0001-7138-9023 https://orcid.org/0000-0002-0122-9523 Fateme Montazeri https://orcid.org/0000-0002-7739-5982 https://orcid.org/0000-0002-3410-5404

\section{Author contributions}

Conceptualization: SH, ART. SB. Data curation: FF, SH, MB, AMM. Formal analysis: HRA, MR, FF, SH. Funding acquisition: ART, SH. Methodology: SH, FF, FM, HRA. Project administration: SH, MB, SB. Visualization: MR, FM, SH, AMM. Writing - original draft: all authors. Writing - review \& editing: all authors. 


\section{References}

1. Zhu Y, Lu H, Huo Z, Ma Z, Dang J, Dang W, et al. MicroRNA-16 inhibits feto-maternal angiogenesis and causes recurrent spontaneous abortion by targeting vascular endothelial growth factor. Sci Rep 2016;6:35536.

2. Lamont K, Scott NW, Jones GT, Bhattacharya S. Risk of recurrent stillbirth: systematic review and meta-analysis. BMJ 2015;350: h3080.

3. Cao X, Cui Y, Zhang X, Lou J, Zhou J, Wei R. The correlation of sperm morphology with unexplained recurrent spontaneous abortion: a systematic review and meta-analysis. Oncotarget 2017;8:55646-56.

4. Zidi-Jrah I, Hajlaoui A, Mougou-Zerelli S, Kammoun M, Meniaoui I, Sallem A, et al. Relationship between sperm aneuploidy, sperm DNA integrity, chromatin packaging, traditional semen parameters, and recurrent pregnancy loss. Fertil Steril 2016;105: 58-64.

5. McQueen DB, Zhang J, Robins JC. Sperm DNA fragmentation and recurrent pregnancy loss: a systematic review and metaanalysis. Fertil Steril 2019;112:54-60.

6. Corzett M, Mazrimas J, Balhorn R. Protamine 1: protamine 2 stoichiometry in the sperm of eutherian mammals. Mol Reprod Dev 2002;61:519-27.

7. Carrell DT, Liu L. Altered protamine 2 expression is uncommon in donors of known fertility, but common among men with poor fertilizing capacity, and may reflect other abnormalities of spermiogenesis. J Androl 2001;22:604-10.

8. Carrell DT, Emery BR, Hammoud S. Altered protamine expression and diminished spermatogenesis: what is the link? Hum Reprod Update 2007;13:313-27.

9. Venkatesh S, Kumar R, Deka D, Deecaraman M, Dada R. Analysis of sperm nuclear protein gene polymorphisms and DNA integrity in infertile men. Syst Biol Reprod Med 2011;57:124-32.

10. Nanassy L, Liu L, Griffin J, Carrell DT. The clinical utility of the protamine 1/protamine 2 ratio in sperm. Protein Pept Lett 2011; 18:772-7.

11. Aoki VW, Liu L, Carrell DT. Identification and evaluation of a novel sperm protamine abnormality in a population of infertile males. Hum Reprod 2005;20:1298-306.

12. Steger K, Wilhelm J, Konrad L, Stalf T, Greb R, Diemer T, et al. Both protamine- 1 to protamine- 2 mRNA ratio and Bcl 2 mRNA content in testicular spermatids and ejaculated spermatozoa discriminate between fertile and infertile men. Hum Reprod 2008; 23:11-6.

13. Jiang W, Sun H, Zhang J, Zhou Q, Wu Q, Li T, et al. Polymorphisms in Protamine 1 and Protamine 2 predict the risk of male infertili- ty: a meta-analysis. Sci Rep 2015;5:15300.

14. Aoki VW, Christensen GL, Atkins JF, Carrell DT. Identification of novel polymorphisms in the nuclear protein genes and their relationship with human sperm protamine deficiency and severe male infertility. Fertil Steril 2006;86:1416-22.

15. Hanzlikova J, Ulcova-Gallova Z, Malkusova I, Sefrna F, Panzner P. $\mathrm{TH} 1-\mathrm{TH} 2$ response and the atopy risk in patients with reproduction failure. Am J Reprod Immunol 2009;61:213-20.

16. Balhorn R, Reed S, Tanphaichitr N. Aberrant protamine 1/protamine 2 ratios in sperm of infertile human males. Experientia 1988;44:52-5.

17. Balhorn R, Corzett M, Mazrimas J, Watkins B. Identification of bull protamine disulfides. Biochemistry 1991;30:175-81.

18. Agarwal A, Bui AD. Oxidation-reduction potential as a new marker for oxidative stress: correlation to male infertility. Investig Clin Urol 2017;58:385-99.

19. Pahune PP, Choudhari AR, Muley PA. The total antioxidant power of semen and its correlation with the fertility potential of human male subjects. J Clin Diagn Res 2013;7:991-5.

20. Rafiee B, Morowvat MH, Rahimi-Ghalati N. Comparing the effectiveness of dietary vitamin C and exercise interventions on fertility parameters in normal obese men. Urol J 2016;13:2635-9.

21. Sheweita SA, Tilmisany AM, Al-Sawaf H. Mechanisms of male infertility: role of antioxidants. Curr Drug Metab 2005;6:495-501.

22. Padayatty SJ, Levine M. Vitamin C: the known and the unknown and Goldilocks. Oral Dis 2016;22:463-93.

23. Aitken J, Fisher H. Reactive oxygen species generation and human spermatozoa: the balance of benefit and risk. Bioessays 1994;16:259-67.

24. Gual-Frau J, Abad C, Amengual MJ, Hannaoui N, Checa MA, Ribas-Maynou J, et al. Oral antioxidant treatment partly improves integrity of human sperm DNA in infertile grade I varicocele patients. Hum Fertil (Camb) 2015;18:225-9.

25. Menkveld R. Clinical significance of the low normal sperm morphology value as proposed in the fifth edition of the WHO Laboratory Manual for the Examination and Processing of Human Semen. Asian J Androl 2010;12:47-58.

26. Pullar JM, Carr AC, Bozonet SM, Rosengrave P, Kettle AJ, Vissers MC. Elevated seminal plasma myeloperoxidase is associated with a decreased sperm concentration in young men. Andrology 2017;5:431-8.

27. Ghasemzadeh J, Talebi AR, Khalili MA, Fesahat F, Halvaei I, Nabi A, et al. Sperm parameters, protamine deficiency, and apoptosis in total globozoospermia. Iran J Reprod Med 2015;13:495-502.

28. Talebi AR, Ghasemzadeh J, Khalili MA, Halvaei I, Fesahat F. Sperm chromatin quality and DNA integrity in partial versus total globozoospermia. Andrologia 2018;50:e12823. 
29. Anbara H, Shahrooz R, Razi M, Malekinejad H, Najafi G. The effect of vitamin $\mathrm{C}$ on mice hemolytic anemia induced by phenylhydrazine: an animal model study using histological changes in testis, pre-implantation embryo development, and biochemical changes. Iran J Basic Med Sci 2018;21:668-77.

30. Gil-Villa AM, Cardona-Maya W, Agarwal A, Sharma R, Cadavid A. Role of male factor in early recurrent embryo loss: do antioxidants have any effect? Fertil Steril 2009;92:565-71.

31. Ko EY, Sabanegh ES Jr. The role of over-the-counter supplements for the treatment of male infertility: fact or fiction? J Androl 2012; 33:292-308.

32. Akmal M, Qadri JQ, AI-Waili NS, Thangal S, Haq A, Saloom KY. Improvement in human semen quality after oral supplementation of vitamin C. J Med Food 2006;9:440-2.

33. Okon UA, Utuk II. Ascorbic acid treatment elevates follicle stimulating hormone and testosterone plasma levels and enhances sperm quality in albino Wistar rats. Niger Med J 2016;57:31-6.

34. Patil PS, Humbarwadi RS, Patil AD, Gune AR. Immature germ cells in semen: correlation with total sperm count and sperm motility. J Cytol 2013;30:185-9.

35. Rahiminia T, Yazd EF, Fesahat F, Moein MR, Mirjalili AM, Talebi AR. Sperm chromatin and DNA integrity, methyltransferase mRNA levels, and global DNA methylation in oligoasthenoteratozoospermia. Clin Exp Reprod Med 2018;45:17-24.

36. Bianchi PG, Manicardi GC, Bizzaro D, Bianchi U, Sakkas D. Effect of deoxyribonucleic acid protamination on fluorochrome staining and in situ nick-translation of murine and human mature spermatozoa. Biol Reprod 1993;49:1083-8.

37. Iranpour FG, Nasr-Esfahani MH, Valojerdi MR, al-Taraihi TM. Chromomycin A3 staining as a useful tool for evaluation of male fertility. J Assist Reprod Genet 2000;17:60-6.

38. Deemeh MR, Tavalee M, Razavi S, Esfahani MH. Evaluation of protamine deficiency and DNA fragmentation in two globozoospermia patients undergoing ICSI. Int J Fertil Steril 2007;1:85-8.

39. Ghasemnejad-Berenji M, Ghazi-Khansari M, Yazdani I, Nobakht M, Abdollahi A, Ghasemnejad-Berenji $\mathrm{H}$, et al. Effect of metformin on germ cell-specific apoptosis, oxidative stress and epididymal sperm quality after testicular torsion/detorsion in rats. Andrologia 2018;50:e12846.

40. Aoki VW, Moskovtsev SI, Willis J, Liu L, Mullen JB, Carrell DT. DNA integrity is compromised in protamine-deficient human sperm. J Androl 2005;26:741-8.

41. Halliwell B, Gutteridge JM. Free radicals in biology and medicine. Oxford: Oxford University Press; 2015.

42. Khavarimehr M, Nejati V, Razi M, Najafi G. Ameliorative effect of omega-3 on spermatogenesis, testicular antioxidant status and preimplantation embryo development in streptozotocin-induced diabetes in rats. Int Urol Nephrol 2017;49:1545-60.

43. Oosterhuis GJ, Mulder AB, Kalsbeek-Batenburg E, Lambalk CB, Schoemaker J, Vermes I. Measuring apoptosis in human spermatozoa: a biological assay for semen quality? Fertil Steril 2000; 74:245-50.

44. Scott L. Pronuclear scoring as a predictor of embryo development. Reprod Biomed Online 2003;6:201-14.

45. Rogenhofer N, Ott J, Pilatz A, Wolf J, Thaler CJ, Windischbauer L, et al. Unexplained recurrent miscarriages are associated with an aberrant sperm protamine mRNA content. Hum Reprod 2017; 32:1574-82.

46. Torregrosa N, Dominguez-Fandos D, Camejo MI, Shirley CR, Meistrich ML, Ballesca JL, et al. Protamine 2 precursors, protamine 1/protamine 2 ratio, DNA integrity and other sperm parameters in infertile patients. Hum Reprod 2006;21:2084-9.

47. Tavalaee M, Razavi S, Nasr-Esfahani MH. Influence of sperm chromatin anomalies on assisted reproductive technology outcome. Fertil Steril 2009;91:1119-26.

48. Mengual L, Ballesca JL, Ascaso C, Oliva R. Marked differences in protamine content and P1/P2 ratios in sperm cells from percoll fractions between patients and controls. J Androl 2003;24:43847.

49. Agarwal A, Aponte-Mellado A, Premkumar BJ, Shaman A, Gupta $S$. The effects of oxidative stress on female reproduction: a review. Reprod Biol Endocrinol 2012;10:49.

50. Pasqualotto EB, Agarwal A, Sharma RK, Izzo VM, Pinotti JA, Joshi $\mathrm{NJ}$, et al. Effect of oxidative stress in follicular fluid on the outcome of assisted reproductive procedures. Fertil Steril 2004;81: 973-6.

51. Greco E, lacobelli M, Rienzi L, Ubaldi F, Ferrero S, Tesarik J. Reduction of the incidence of sperm DNA fragmentation by oral antioxidant treatment. J Androl 2005;26:349-53.

52. Tremellen K. Treatment of sperm oxidative stress: a collaborative approach between clinician and embryologist. In: Henkel R, Samanta L, Agarwal A, eds. Oxidants, antioxidants and impact of the oxidative status in male reproduction. London: Academic Press; 2019. p. 225-35.

53. Vani K, Kurakula M, Syed R, Alharbi K. Clinical relevance of vitamin $C$ among lead-exposed infertile men. Genet Test Mol Biomarkers 2012;16:1001-6.

54. Yun SJ, Bae GS, Park JH, Song TH, Choi A, Ryu BY, et al. Antioxidant effects of cultured wild ginseng root extracts on the male reproductive function of boars and guinea pigs. Anim Reprod Sci 2016;170:51-60. 